\title{
Cattle rob(1;29) originating from complex chromosome rearrangements as revealed by both banding and FISH-mapping techniques`
}

\author{
G. P. Di Meo ${ }^{1}$, A. Perucatti ${ }^{1}$, R. Chaves $^{2}$, F. Adega ${ }^{2}$, L. De Lorenzi ${ }^{3}$, L. Molteni ${ }^{3}$, A. De Giovanni ${ }^{3}$, \\ D. Incarnato ${ }^{1}, \mathrm{H}$. Guedes-Pinto ${ }^{2}, \mathrm{~A}$. Eggen $^{4} \&$ L. Iannuzzi ${ }^{1 *}$ \\ ${ }^{1}$ National Research Council (CNR), ISPAAM, CNR-ISPAAM, Laboratory of Animal Cytogenetics and Gene \\ Mapping, Via Argine 1085, 80147 Naples, Italy; Tel: +39-0815964977; Fax: +39-0815965291; \\ E-mail: L.Iannuzzi@iabbam.na.cnr.it; ${ }^{2}$ Department of Genetics and Biotechnology, Center of Genetics and \\ Biotechnology of the University of Trás-os-Montes and Alto Douro (CGB-UTAD), Vila Real, Portugal; \\ ${ }^{3}$ Animal Production Institute, Agricultural Faculty of Sciences, Milan, Italy; ${ }^{4}$ Department of Animal Genetics, \\ Laboratory of Biochemical Genetics and Cytogenetics, INRA, Jouy-en-Josas, France \\ * Correspondence
}

Key words: cattle, evolution, fluorescence in-situ hybridization, satellite DNA, translocation

\begin{abstract}
Sixteen carriers of rob(1;29) (one of which was homozygous) from six different breeds (four Italian and two Portuguese), two heterozygous carriers of rob(26;29), three river buffaloes and two sheep were cytogenetically investigated in this study by using banding and FISH-mapping techniques (the latter only in cattle and river buffalo). Single- and dual-colour FISH were used with bovine probes containing both INRA143 (mapping proximally to BTA29) and bovine satellite (SAT) DNA SAT I, SAT III and SAT IV (mapping at the centromeric regions of cattle chromosomes). The combined use of these probes, the comparison of rob(1;29) with the dicentric $\operatorname{rob}(26 ; 29)$ and with both river buffalo and sheep chromosomes (biarmed pairs) allowed us to hypothezise that $\operatorname{rob}(1 ; 29)$ originated from complex chromosomal rearrangements through at least three sequential events: (a) centric fusion with the formation of a dicentric chromosome; (b) formation of a monocentric chromosome with loss of SAT I from both BTA1 and BTA29, most of SAT IV from BTA29 and, probably, some repeats of SAT III from BTA1; (c) double pericentric inversion or, more probably, a chromosome transposition of a small chromosome segment containing INRA143 from proximal p-arms to proximal q-arm of the translocated chromosome.
\end{abstract}

\section{Introduction}

Domestic animal cytogenetics was born when the first cattle chromosome (BTA) abnormality Y rob(1;29) Y was discovered (Gustavsson \& Rockborn 1964) and,

\footnotetext{
This study is dedicated to Professor Ingemar Gustavsson, a teacher for many of us, recently retired.
}

more specifically, when its deleterious effect on the fertility of Swedish red cattle was demonstrated (Gustavsson 1969, Dyrendhal \& Gustavsson 1979). These studies have attracted the attention of many cytogenetic laboratories and several chromosome abnormalities have been discovered in many domestic species (Gustavsson 1980). However, Robertsonian translocations (i.e. centric fusions $=\mathrm{CF}$ ) have 
been the most common chromosome abnormalities found in cattle (Gustavsson 1980, Long 1985, Iannuzzi et al. 1987). All these translocations have been found to be dicentric (two centromeres), except for $\operatorname{rob}(1 ; 29)$ which is monocentric. Dicentric translocations are to be considered of recent origin as chromosome mutations, as they have been found occasionally in several animals. By contrast, $\operatorname{rob}(1 ; 29)$ is considered to be of ancient origin because it has been found in more than 40 different breeds (Popescu \& Pech 1991), mainly meat breeds, with different frequencies, reaching the highest in Portuguese Barrosã cattle, with $70 \%$ of individuals with $\operatorname{rob}(1 ; 29)(17 \%$ of which are homozygous) (RangelFigueiredo \& Iannuzzi 1993). Only in the Italian Grey Alpine breed has an appreciable frequency (11\%) of carriers been found to carry a dicentric CF involving chromosomes 26 and 29 (Di Meo et al. 2000, Iannuzzi et al. 2001). CF has also been the most common chromosome rearrangement occurring during the autosome karyotype evolution of bovids (reviewed in Iannuzzi \& Di Meo 1995).

$\operatorname{Rob}(1 ; 29)$ has been characterized by using C-, Gand R-banding techniques. These approaches revealed that $\operatorname{rob}(1 ; 29)$ is monocentric with a large block of heterochromatin located proximally to the q-arms (Iannuzzi et al. 1987, Rangel-Figueiredo \& Iannuzzi 1993). The comparison between $\operatorname{rob}(1 ; 29)$ and both BTA1 and BTA29 revealed that the centromere of BTA29 may be lost and that of BTA1 retained (Iannuzzi et al. 1987, Di Meo et al. 1990). However, the proximal region on $\operatorname{rob}(1 ; 29)$ q-arms appeared larger than the corresponding one of BTA1, suggesting that part of the BTA29 material (centromere) could be retained on the q-arm during the centric fusion event (Iannuzzi et al. 1987, Di Meo et al. 1990). Lack of complete pairing between the translocated chromosome and normal BTA1 and BTA29 has also been demonstrated by synaptonemal complex analysis (Switonski et al. 1987).

A further contribution to understanding the origin of this translocation was achieved when Eggen et al. (1994) used a probe (INRA143) mapping proximally to BTA29 and proximally to $\operatorname{rob}(1 ; 29)$ q-arms, suggesting that this translocation may have originated both from a centric fusion and a pericentric inversion. However, they investigated only two animals from the same breed.

More recently, centromeric probes containing cattle satellites (SAT) DNA I, III and IV were hybridized to five different Portuguese cattle carriers of $\operatorname{rob}(1 ; 29)$ from two breeds by using a dual-colour FISH (Chaves et al. 2003). On the basis of this approach the same authors suggested an alternative origin for this translocation such as reciprocal translocations and loss of SAT. In particular, there was a retention of some repetitive sequences from the BTA29 centromere on the $\operatorname{rob}(1 ; 29) \mathrm{p}$-arms, and from BTA1 on $\operatorname{rob}(1 ; 29)$ q-arm side.

In this study we investigated 16 carriers (one of which was a homozygous carrier) from six different breeds (four Italian and two Portuguese), and combined both banding and dual-colour FISH-techniques using probes containing both INRA143 and bovine satellites DNA SAT I, SAT III and SAT IV. Furthermore, comparison with other species, such as river buffalo (BBU) and sheep (OAR), which are thought to evolve their karyotypes through $\mathrm{CF}$ and with the dicentric $\operatorname{rob}(26 ; 29)$ of the Italian Grey Alpine cattle breed (Di Meo et al. 2000, Iannuzzi et al. 2001), allowed us to confirm the loss of SAT as reported earlier (Chaves et al. 2003) and support different and complex chromosome rearrangements originating $\operatorname{rob}(1,29)$.

\section{Material and methods}

Sixteen rob(1;29) carriers, from six different cattle breeds comprising four Italian (Podolian Y two animals, Chianina Y four animals, Marchigiana Y five animals, Maremmana Y three animals) and two Portuguese breeds (Barrosã Y two animals including one homozygous carrier, Alentejana Y one animal), two heterozygous carriers of $\operatorname{rob}(26 ; 29)$ from the Grey Alpine breed, three river buffaloes and two sheep were studied. Peripheral blood lymphocytes cultures, CBA- and R-banding, as well as FISH mapping technique were as previously reported (Iannuzzi 2003, Di Meo et al. 2005).

As probes we used a bovine BAC clone containing INRA143 (Eggen et al. 1994) and bovine satellites DNA (SAT) I, III and IV previously employed by Chaves et al. (2003). SAT I, III and IV probes were labelled with both biotin or digoxigenin (Chaves et al. 2003), while INRA143 was only biotin-labelled by nick-translation, and ethanol-precipitated in the presence of bovine Cot-1 DNA for the in-situ suppression of repetitive sequences. At least 15 meta- 
phases per animal were captured by a colourcoupled CCD camera (Coolsnap, Photometrics) and later processed by superimposing FITC (green) or/and rhodamine (red) signals on RBHbanded chromosomes. R-banded ideograms of BTA1, BTA29 and $\operatorname{rob}(1 ; 29)$ were constructed on the basis of the latest cattle standard chromosome nomenclature (ISCNDB2000 2001).

\section{Results and discussion}

Figure 1 shows CBA-banding patterns of cattle $\operatorname{rob}(1 ; 29)$ compared with those of both river buffalo and sheep. While a large block of heterochromatin can be seen on the proximal region of $\operatorname{rob}(1 ; 29)$ q-arms, the biarmed pairs of both river buffalo and sheep (originating from the centric fusion translocation of 10 and six cattle/goat homoeologues, respectively) show small C-bands. However, the intensity of staining (fluorescence) was brighter in the normal autosomes than that achieved on the $\operatorname{rob}(1 ; 29)$ heterochromatin block. In the same figure, details of $\operatorname{rob}(1 ; 29)$ with INRA143 FITC-signals on heterozygous (Figure 1D) and homozygous (Figure 1E) carriers show very clearly that INRA143 maps in the pericentromeric region of BTA29 and proximally to $\operatorname{rob}(1 ; 29)$ q-arms. Since the same results were obtained in all the carriers investigated from six different breeds, we believe that this pattern is common to all $\operatorname{rob}(1 ; 29)$ carriers as an ancestral rearrangement. Interestingly, propidium iodide (PI) used for chromosome staining in single-colour FISHapplications (Figure 1D,E) stains intensely the centromeres of all autosomes and only partially (small positive region) that of $\operatorname{rob}(1 ; 29)$.

Co-hybridization of INRA143 and SAT I on RBH- banded chromosomes shows very clearly that SAT I is present in both normal BTA1 and BTA29 and apparently absent from both arms of $\operatorname{rob}(1,29)$ (Figure 2a). SAT IV is largely present on BTA29, in a small amount on proximal $\operatorname{rob}(1 ; 29)$ p-arms, and is lacking on both normal BTA1 and $\operatorname{rob}(1 ; 29)$ q-arms (Figure 2b). SAT I and SAT IV seem to overlap on BTA29. Indeed, both SAT hybridizations cover the same areas which are very close to INRA143 FITC-signals (Figure 2a,b).
SAT III is lacking in BTA29 and present in BTA1, together with SAT I, although the latter appears more centromeric to SAT III (Figure 2c), as previously reported (Chaves et al. 2003). SAT III is the only SAT present on $\operatorname{rob}(1 ; 29)$ q-arms (Figure 2d), and cohybridization with INRA143 reveals that the large area highlighted by SAT III should also include the chromosome region containing INRA143 (Figure 2d). This large area containing both SAT III and INRA143 chromosome region agrees perfectly with the Cbanding patterns of this translocation (Figure 1A). The loss of SAT I during the formation of $\operatorname{rob}(1 ; 29)$ might also explain the less intense C-band of $\operatorname{rob}(1 ; 29) \mathrm{HC}$ block (Figure 1), when compared with the remaining autosomes containing SAT I (this study; Chaves et al. 2003). The same can be observed in Figure 1D,E when comparing the less-stained centromere of $\operatorname{rob}(1 ; 29)$ with the remaining acrocentric chromosomes, when using PI-staining.

Unlike rob(1;29), SAT I and SAT IV are both present in $\operatorname{rob}(26 ; 29)$ (Figure 2e), suggesting that all SAT are probably conserved in dicentric translocations, although this finding needs further confirmation on other $\mathrm{CF}$ analysis.

BBU5 originated by centric fusion of homoeologues to BTA16 and BTA29 (q- and p-arms, respectively) (Iannuzzi et al. 1990, CSKBB 1994), and C-bands are very small in this chromosome (Figure 1B). When hybridizing INRA143 on river buffalo chromosomes, clear signals were detected on BBU5, close to the centromere but proximally to the p-arms (Figure 2f), confirming that almost all the heterochromatin (i.e. SAT) was lost during, before or after the centric fusion event that produced this biarmed chromosome (as with the others of this species) (Figure 1B), and apparently no further rearrangements occurred after the formation of this chromosome.

On the basis of these observations we hypothesize that $\operatorname{rob}(1 ; 29)$ of cattle may have originated from at least three sequential events (Figure 3):

1. Centric fusion with the formation of a dicentric chromosome $(1+29)$ (Figure 3C) as occurred on $\operatorname{rob}(26 ; 29)$ and, very probably, in all dicentric Robertsonian translocations;

2. The origin of a monocentric chromosome with loss of a chromosome fragment containing SAT I from 
both BTA1 and BTA29, most SAT IV repeats from BTA29 and, probably, some SAT III repeats from BTA1 (Figure 3D). However, after this event, the small chromosome region containing INRA143 remained in the p-arms (Figure 3D), while we know that this region is located proximally to $\operatorname{rob}(1 ; 29) \mathrm{q}-$ arms (Figures 1 and 2). Starting from this monocentric translocation, two possible and alternative chromosome rearrangements took place to reach the present rob(1;29): (a) A double pericentric inversion: the first with two breaks occurring on the centromere of $\operatorname{rob}(1 ; 29)$ and proximally to the p-arms (containing a chromosome region where both INRA143 and the rest of SAT IV are present); the second with two breaks occurring on the centromere of $\operatorname{rob}(1 ; 29)$ and proximally to the q-arms containing a small region with SAT IV which, from proximal q-arms, returns to proximal p-arms to stabilize the chromosome (Figure 3EYG). (b) Transposition of a small chromosome region containing INRA143 from proximal p-arms to proximal q-arms in the pericentromeric region containing SAT III (Figure 3H,I).

Transposition is a complex chromosome rearrangement which requires at least three chromosome breaks. However, these rearrangements have occurred not only in primates (Montefalcone et al. 1999, Ventura et al. 2004) but also in bovids, essentially in the X-chromosomes (Robinson et al. 1998, Iannuzzi et al. 2000). Hence, we incline towards the transpo- sition hypothesis rather than double pericentric inversion. Indeed, while double pericentric inversion would exclude SAT III at the proximal q-arm pericentromeric region (due to the presence of the small chromosome region containing INRA143) (Figure 3G), with the transposition event the chromosome region containing INRA143 has been included in the pericentromeric region containing SAT III (Figure 3I). Certainly, the presence of SAT at the centromere region gives to the fused chromosome the necessary stability for being maintained. This hypothesis is also in agreement with the $\mathrm{C}$ - banding patterns achieved in $\operatorname{rob}(1 ; 29)$ (Figure 1A) where the $\mathrm{C}$-banding staining covers the whole proximal q-arm region (SAT III) and a very small proximal p-arm region (SAT IV).

Although $\operatorname{rob}(1 ; 29)$ was discovered more than 40 years ago, and is found worldwide, its origin still remains unclear. The present study, in addition to previous findings (Eggen et al. 1994, Chaves et al. 2003), shows that complex chromosome rearrangements such as (a) CF with the formation of dicentric and subsequent monocentric chromosomes with (b) loss of SAT followed by (c) pericentric inversions or, more probably, (d) by a transposition event, occurred during the formation of this famous chromosome polymorphism.
Comparison of this translocation with other biarmed chromosomes of species for which $\mathrm{CF}$ is a prevalent mechanism in their autosomal chromosome evolution (river buffalo and sheep) and where very small amounts of HC were detected, suggests that $\operatorname{rob}(1 ; 29)$ is still evolving by progressive loss of $\mathrm{HC}$ from q-arms (SAT III).

\section{Acknowledgements}

The authors thank Mr A. Navarro, CNR-ISPAAM of Naples, Italy, for excellent technical assistance.

\section{References}

Chaves R, Adega F, Heslop-Harrison JS, Guedes-Pinto H, Wienberg J (2003) Complex satellite DNA reshuffling in the polymorphic $\mathrm{t}(1 ; 29)$ Robertsonian translocation and evolutionarily derived chromosomes in cattle. Chromosome Res 11: 641 Y 648 .

CSKBB (1994) Standard karyotype of the river buffalo (Bubalus bubalis L., $2 \mathrm{n}=50$ ). Report of the Committee for the Stan- dardization of Banded Karyotypes of the River Buffalo (L. Iannuzzi, coordinator). Cytogenet Cell Genet 67: 102Y113.

Di Meo GP, Iannuzzi L, Perucatti A, Ferrara L (1990) Chromosome markers in cattle revealed by high resolution $\mathrm{G}+\mathrm{C}$-bands. Caryologia 43: 149Y156.

Di Meo GP, Molteni L, Perucatti A et al. (2000) Chromosomal characterization of three centric fusion translocations in cattle using G-, R- and C-banding and FISH technique. Caryologia 53: $213 Y 218$

Di Meo GP, Perucatti A, Floriot S et al. (2005) Chromosome evolution and improved cytogenetic maps of the Y chromosome in cattle, zebu, river buffalo, sheep and goat. Chromosome Res 13: 349 Y355.

Dyrendhal I, Gustavsson I (1979) Sexual functions, semen characteristics and fertility of bulls carrying the 1/29 chromosome translocation. Hereditas 90: 281 Y289.

Eggen A, Oustry A, Vaiman D, Ferretti L, Fries R, Cribiu EP (1994) Bovine syntenic group U7, previously assigned to Gbanded chromosome 25 in the ISCNDA nomenclature, assigns to R-banded chromosome 29. Hereditas 121: 295 Y300.

Gustavsson I (1969) Cytogenetics, distribution and phenotypic effects of a translocation in Swedish cattle. Hereditas 63: 68Y169.

Gustavsson I (1980) Chromosome aberrations and their influence on the reproductive performance of domestic animals $Y$ a review. Z Tierzuchtg Zuchtgsbiol 97: 176Y195.

Gustavsson I, Rockborn G (1964) Chromosome abnormality in three cases of lymphatic leukaemia in cattle. Nature 203: 990.

Iannuzzi L (2003) Methodologies applied on domestic animal chromosomes. In Sgaramella V, Eridani S, eds., Methods in Molecular Biology, vol. 240: Artificial Mammalian Chromosomes. Totowa, NJ: Humana Press, pp. 15 Y34.

Iannuzzi L, Di Meo GP (1995) Chromosomal evolution in bovids: a comparison of cattle, sheep and goat G- and R-banded chromo somes and cytogenetic divergences among cattle, goat and river buffalo sex chromosomes. Chromosome Res 3: 291Y 299. 
Iannuzzi L, Di Berardino D, Gustavsson I, Ferrara L, Di Meo GP (1987) Centromeric loss in translocations of centric fusion type in cattle and water buffalo. Hereditas 106: 73 Y81.

Iannuzzi L, Di Meo GP, Perucatti A, Ferrara L (1990) The high resolution G- and R-banding patterns in chromosomes of river buffalo (Bubalus bubalis L.). Hereditas 112: 209 Y215.

Iannuzzi L, Di Meo GP, Perucatti A, Incarnato D, Schibler L, Cribiu EP (2000) Comparative FISH-mapping of bovid X chromosomes reveals homologies and divergences between the subfamilies Bovinae and Caprinae. Cytogenet Cell Genet 89:171Y176.

Iannuzzi L, Di Meo GP, Perucatti A et al. (2001) A new balanced autosomal reciprocal translocation in cattle revealed by banding techniques and HSA-painting probes. Cytogenet Cell Genet 94:225Y228.

ISCNDB2000 (2001) International System for Chromosome Nomenclature of Domestic Bovids. Di Berardino D, Di Meo GP, Gallagher DS, Hayes H, Iannuzzi L (co-ordinator), eds. Cytogenet Cell Genet 92: 283Y299.

Long S (1985) Centric fusion translocations in cattle: a review. Vet Rec 116: 516Y518.

Montefalcone G, Tempesta S, Rocchi M, Arcidiacono N (1999) Centromere repositioning. Genome Res 9: 1184Y1188.

Popescu CP, Pech A (1991) Une bibliographie sur la translocation 1/29 de bovins dans le monde (1964Y1990). Ann Zootech 40: 271 Y305.

Rangel-Figueiredo T, Iannuzzi L (1993) Frequency and distribution of $\operatorname{rob}(1 ; 29)$ in three Portuguese cattle breeds. Hereditas 119: 233 Y 237.

Robinson TJ, Harrison WR, Ponce de Leon FA, Davis SK, Elder FFB (1998) A molecular cytogenetic analysis of the X chro- mosome repatterning in the Bovidae: transpositions, inversions, and phylogenetic inference. Cytogenet Cell Genet 80: 179 Y 184.

Switonski M, Gustavsson I, Ploen L (1987) The nature of the 1;29 translocation in cattle as revealed by synaptonemal complex analysis using electron microscopy. Cytogenet Cell Genet 44: 103Y 111.

Ventura M, Weigl S, Carbone L et al. (2004) Recurrent sites for new centromere seeding. Genome Res 14: 1696Y1703. 

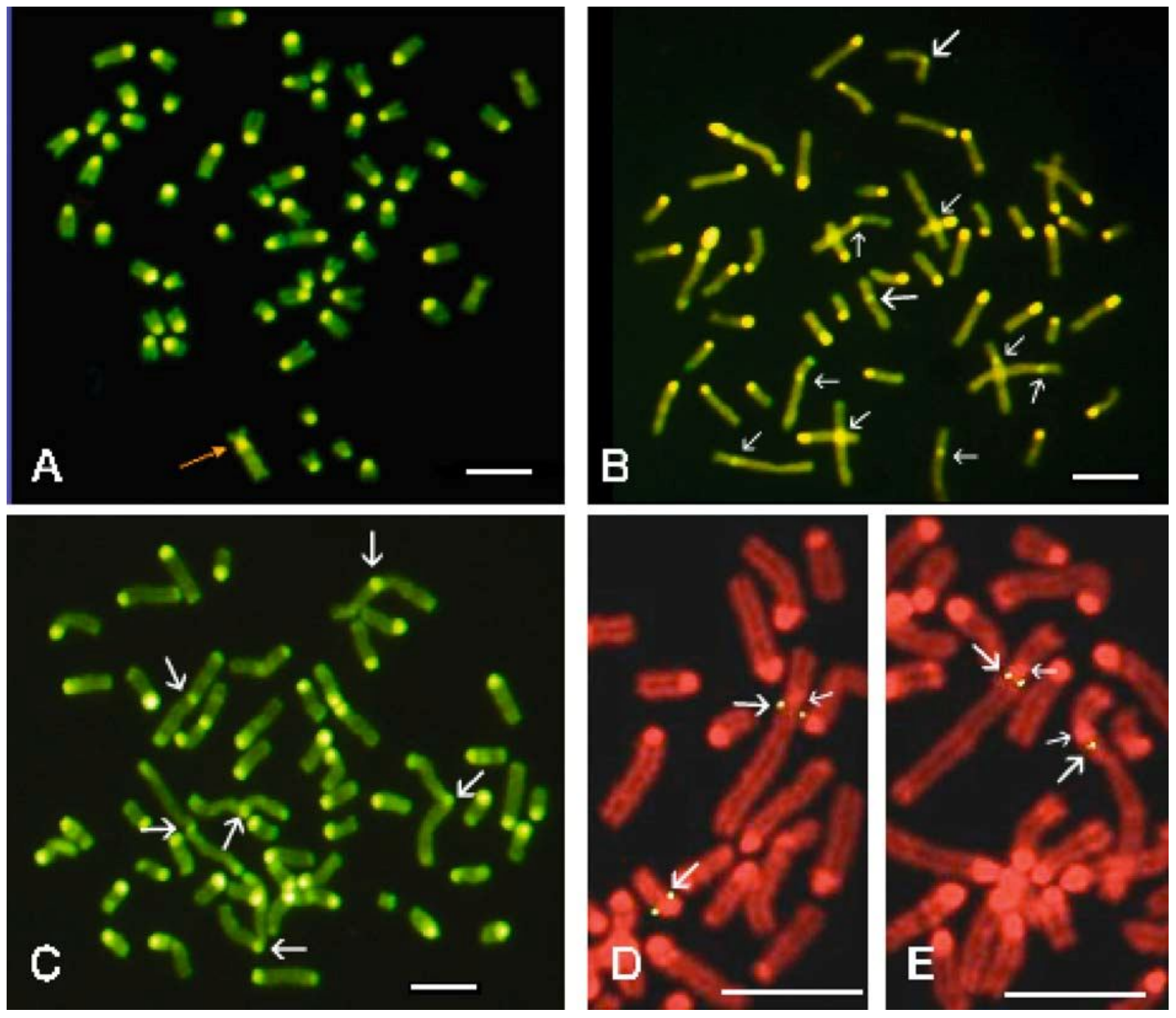

Figure 1. A: CBA-banding patterns in a female cattle heterozygous carrier of $\operatorname{rob}(1 ; 29)$ (arrow). Note the large HC-block of the translocated chromosome and its less intense fluorescence compared to that of remaining C-banded autosomes. CBA-banding patterns in river buffalo (B) and sheep (C) chromosomes reveal small C-bands in the biarmed chromosomes (arrows). Larger arrows in B indicate BBU5 \{i.e. rob(16;29)\}. Details of rob(1;29) heterozygous (D) and homozygous (E) carriers treated for FISH with INRA143. Large and small arrows indicate hybridization FITC-signals and centromeres, respectively, on rob(1;29). Bar $=10 \mathrm{~mm}$. 

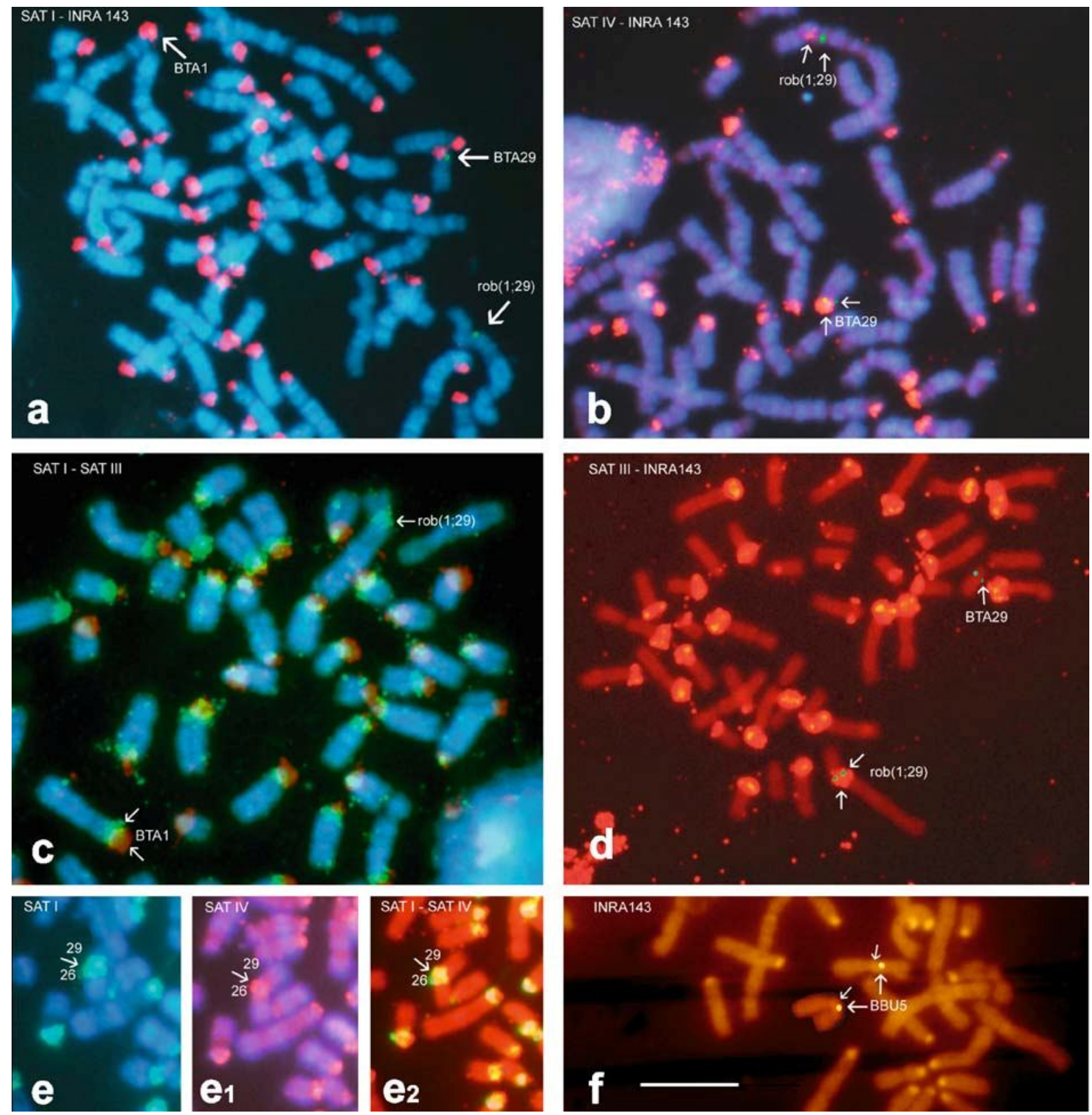

Figure 2. Dual-colour (a, b, c, d, e) and single-colour (f) FISH on metaphases of rob(1;29) (a, b, c, d) and rob(26;29) (e) carriers, as well as on BBU5 (f). The dual-colour FISH has also been combined with RBH-banding in figure a, b, c and e. (a) SAT I signals (red) are present on both BTA1 (identified by RBH-banding) and BTA29 (identified by INRA143) (arrows) and absent on rob (1;29), where only INRA143 signals (green) localized proximal to q-arms can be seen (arrow). (b) SAT IV (red) is present on BTA29 (arrows) and only partially on rob(1;29) proximal p-arms (arrow). (c) The co-hybridization of SAT I (red) and SAT III (green) shows that both SAT are present on BTA1, SAT I being more centromeric than SAT III (arrows), the latter also being present on the proximal q-arms regions of rob(1;29). (d) The co- hybridization of SAT III and INRA143 shows that SAT III is absent on BTA29 and present on the proximal q-arms of rob(1;29) (arrow). (e) The co-hybridization of both SAT I and SAT IV on the dicentric rob(26;29) reveals that both SAT are present in this translocation (arrows). (f) INRA143 maps proximally to BBU5p (large arrows) and very close to the centromere (small arrows). Bar $=10 \mathrm{~mm}$. 


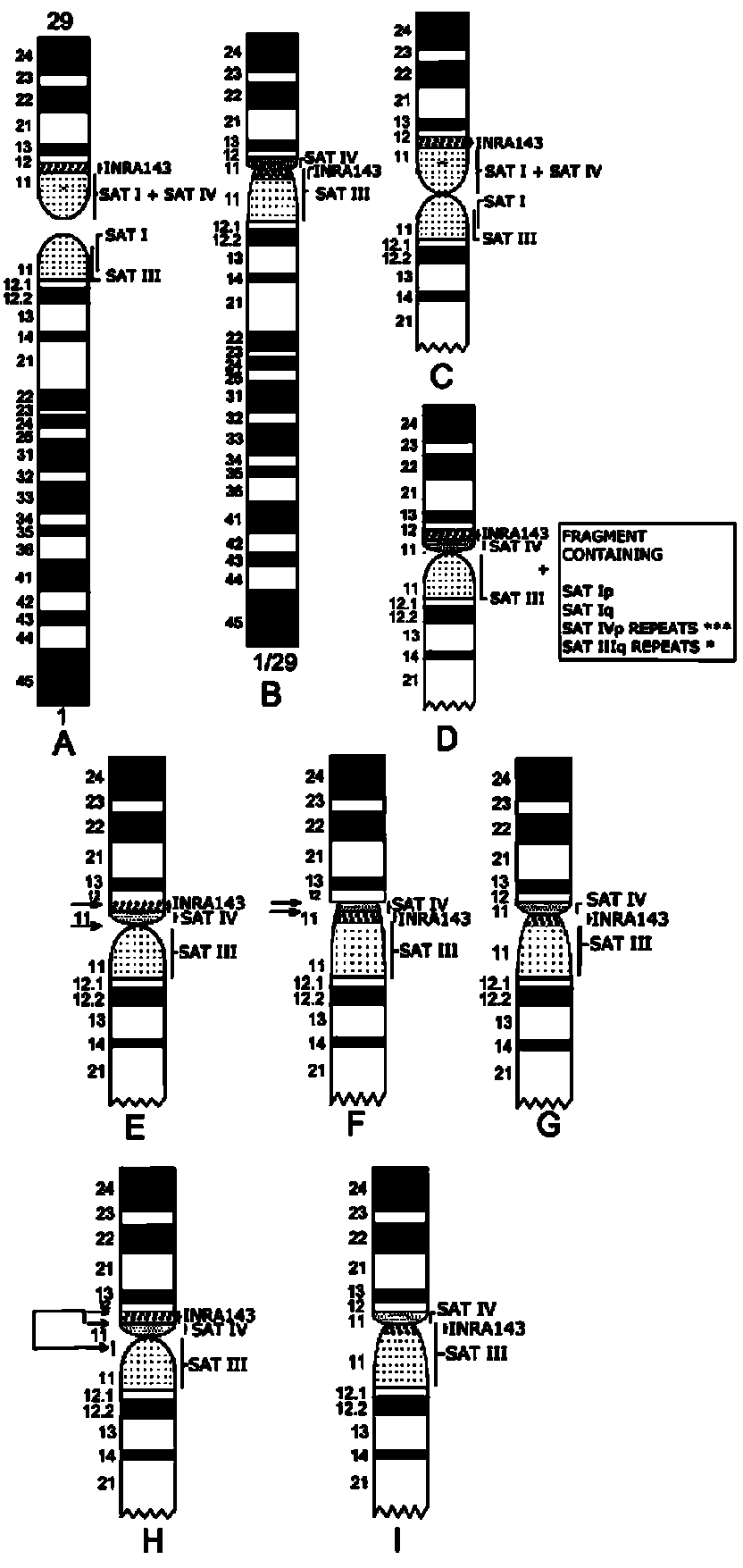

Figure 3. Ideograms of R-banded BTA1 and BTA29 (A) and present rob(1;29) (B). Possible origin of rob(1;29) through the formation of a dicentric (C) and subsequent monocentric (D) chromosome with loss of a chromosome fragment containing SAT I from both p- (BTA29) and q- (BTA1) arms, most of SAT IV repeats from p-arms (BTA29) and, probably, some repeats of SAT III from q-arms (BTA1). Further steps from monocentric rob(1;29) (E,H) to reach the present $\operatorname{rob}(1 ; 29)(\mathrm{G}, \mathrm{I})$ could be a double pericentric inversion $(\mathrm{E}, \mathrm{F})$ or a chromosome transposition of a chromosome fragment containing INRA143 from proximal p-arms to proximal q-arms (H). Ideograms of $\operatorname{rob}(1 ; 29)$ have been cut (DYI) to save space and to better show the rearrangements occurring only in the pericentromeric regions. 